\title{
HUMEDAD EDÁFICA, NITRÓGENO Y HONGOS ECTOMICORRÍZICOS COMESTIBLES EN EL CRECIMIENTO DE PINO
}

\section{SOIL HUMIDITY, NITROGEN AND EDIBLE ECTOMYCORRHIZAL FUNGI ON PINE GROWTH}

\author{
Víctor M. Perea-Estrada, Jesús Pérez-Moreno*, Luis Villarreal Ruiz, Antonio Trinidad Santos, \\ Ma. de Lourdes de la I. de Bauer, Víctor M. Cetina Alcalá y Leonardo Tijerina Chávez
}

Colegio de Postgraduados. Km 36.5 Carretera México-Texcoco. 56230, Montecillo, Texcoco, Estado de México. Tel.595 9520200 ext. 1280.

*Autor para correspondencia (jperezm@colpos.mx)

\section{RESUMEN}

El efecto de la humedad del sustrato y la fertilización nitrogenada en plantas inoculadas con hongos ectomicorrízicos (ECM) ha recibido escasa atención, a pesar de la relevancia ecológica y sensibilidad de estos hongos a factores ambientales. En este estudio se evaluó en invernadero la influencia de dos regímenes de humedad del sustrato [80 a 100 y 40 a $60 \%$ de la capacidad máxima de retención de agua del sustrato (CMRAS)], la adición de tres dosis de nitrógeno mineral (140, 70 y $0 \mathrm{~kg}$ de $N$ ha $\left.^{-1}\right)$ y la inoculación con tres hongos ectomicorrízicos comestibles (Hebeloma spp., Laccaria spp. y Clavulina aff. cinerea), en el desarrollo de dos especies de pinos (Pinus patula Schl. et Cham y Pinus hartwegii Lindl). Se midió altura y diámetro del tallo a 90, 180 y 310 d después de la siembra, número de raíces cortas, pesos secos de parte aérea y raíz, y la colonización ectomicorrízica. Los efectos relevantes $(P \leq 0.05)$ en las plantas fueron originados por la humedad del sustrato y la inoculación con hongos ectomicorrízicos. Las interacciones sobresalientes $(P \leq \mathbf{0 . 0 5})$ fueron las de la humedad del sustrato y la especie de pino, la humedad del sustrato y la inoculación con hongos ECM, y la especie de pino y la inoculación. La altura y el peso seco aumentaron como consecuencia de la inoculación con Hebeloma spp. y Laccaria spp. En las plantas de $\boldsymbol{P}$. patula inoculadas con Hebeloma spp., el régimen de humedad de 80 a $100 \%$ produjo mayor peso seco total $(46 \%)$ y altura $(25 \%)$ que el régimen de humedad de 40 a $60 \%$. En $P$. patula inoculado con Hebeloma spp. y fertilizado con $140 \mathrm{~kg} \mathrm{~N} \mathrm{ha}^{-1}$, se apreció un aumento en el peso seco del follaje (29\%), comparado con el tratamiento sin fertilizante nitrogenado. El crecimiento de $\boldsymbol{P}$. patula y $P$. hartwegii fue afectado diferencialmente en las humedades evaluadas, en función de los hongos ECM inoculados.

Palabras clave: Pinus patula, $P$. hartwegii, hongos ectomicorrízicos, humedad, nitrógeno.

\section{SUMMARY}

The effect of soil moisture and nitrogen fertilization on plants inoculated with ectomycorrhizal fungi has received limited attention, despite the ecological significance and sensitivity of these fungi to environmental factors. In this study the influence of two soil moisture regimes [80 to 100 and 40 to $60 \%$ of the maximum capacity of water retention by the substrate (MCWRS)], the addition of three doses of mineral nitrogen (140, 70 and $0 \mathrm{~kg}$ of $\left.\mathrm{N} \mathrm{ha}^{-1}\right)$ and the inoculation with three edible ectomycorrhizal fungi (Hebeloma spp. Laccaria spp. and
Clavulina aff. cinerea) on the growth of two species of pine (Pinus patula Schl. et Cham and Pinus hartwegii Lindl), was evaluated under greenhouse conditions. Shoot height and diameter at 90, 180 and 310 d after sowing, number of short roots, shoot and root dry weights, and ectomycorrhizal colonization were evaluated. Relevant effects ( $P$ $\leq \mathbf{0 . 0 5})$ on plants were originated by soil moisture and inoculation with ectomycorrhizal fungi. The significant interactions $(P \leq \mathbf{0 . 0 5})$ were those established between soil moisture and pine species, soil moisture and inoculation with ectomycorrhizal fungi, and pine species and inoculation. Plant height and dry weight increased as a result of inoculation with Hebeloma spp. and Laccaria spp. In P. patula inoculated with Hebeloma spp. the soil moisture regime from 80 to $100 \%$ produced higher dry weight $(46 \%)$ and height $(25 \%)$, than the soil moisture regime from 40 to $60 \%$. In P. patula inoculated with Hebeloma spp. and fertilized with $140 \mathrm{~kg} \mathrm{~N} \mathrm{ha}^{-1}$ there was an increase in foliage dry weight $(29 \%)$ compared to the control without nitrogen fertilizer. The growth of $\boldsymbol{P}$. patula y $\boldsymbol{P}$. hartwegii was differentially affected in the evaluated humidity regimes, depending on the inoculated ectomycorrhizal fungi.

Index words: Pinus patula, P. hartwegii, ectomycorrhizal fungi, humidity, nitrogen.

\section{INTRODUCCIÓN}

Los hongos ectomicorrízicos (ECM) forman una interfase entre el suelo y la planta huésped en donde las hifas extrarradicales sirven como extensiones de la raíz en gimnospermas y funcionan como estructuras de absorción de agua y minerales (Leake et al., 2004). El micelio externo de las ectomicorrizas puede ampliar las raíces de las plantas varios metros, explorar en forma más eficiente el suelo y formar agregados, los cuales ayudan a mantener la estructura y la aireación en el suelo (Bogeat-Triboulot et al., 2004). Las micorrizas, además de participar en la absorción de agua y nutrimentos, favorecen cambios fisiológicos y morfológicos de la planta huésped. Estos cambios de la planta, causados por la micorrización, aminoran condiciones de estrés ambiental, como la limitación de agua y nutrimentos (Davies et al., 1996). 
Actualmente, en diversas partes del mundo existe una declinación de los bosques como consecuencia de la contaminación ambiental y del cambio climático (Alvarado-Rosales et al., 2002; Paoletti et al., 2007). Dos factores que afectan conspicuamente a los hongos ectomicorrízicos asociados con los árboles, son el estrés hídrico y el depósito de N. Al respecto, el estrés hídrico reduce la colonización ectomicorrízica (Nilsen et al., 1998), y el depósito de $\mathrm{N}$ atmosférico afecta diferencialmente a las comunidades de hongos ECM. Unas especies de hongos ECM son tolerantes al depósito de N, denominadas nitrofílicas, y otras especies son susceptibles, denominadas nitrofóbicas; en estas últimas se ha registrado declinación en la producción de esporomas (Carfrae et al., 2006).

En México, el estudio de la influencia del estrés hídrico y del depósito de $\mathrm{N}$ en las especies ectomicorrízicas no se ha estudiado, según lo publicado, a pesar de que se ha documentado que en los últimos años ha habido un incremento del depósito de $\mathrm{N}$ atmosférico en las áreas boscosas del centro de México (Fenn et al., 2003). Por ello, el presente trabajo tuvo como objetivo determinar, en condiciones de invernadero, la influencia de dos regímenes de humedad en el sustrato y tres dosis de $\mathrm{N}$ mineral, en la inoculación de tres hongos ECM comestibles (Hebeloma spp., Laccaria spp. y Clavulina aff. cinerea), y su efecto en el crecimiento de dos especies de pinos nativos mexicanos de importancia económica y ecológica, Pinus patula y Pinus hartwegii.

\section{MATERIALES Y MÉTODOS}

El trabajo se hizo en Montecillo, Texcoco, Estado de México, durante los años 2006 y 2007. Para esta investigación se seleccionaron dos especies endémicas de pinos mexicanos de importancia forestal: Pinus patula Schl. et Cham. y Pinus hartwegii Lindl. P. patula se distribuye en la Sierra Madre Oriental, desde Tamaulipas hasta Oaxaca y se extiende también en la Sierra Madre del Sur, hasta el Estado de Guerrero. Más de un millón de hectáreas de $P$. patula se han plantado en el mundo, principalmente en África y Sudamérica (Dvorak et al., 2000). P. hartwegii, conocido como pino de las alturas, en México se localiza en el límite de la vegetación arbórea, a altitudes de 2800 a $4200 \mathrm{~m}$ (Campos, 1993).

Las semillas de $P$. patula y $P$. hartwegii aquí utilizadas proceden de los municipios de Villa Victoria y Zinacantepec, Estado de México, respectivamente. Como sustrato se utilizó una mezcla de suelo, arena y aserrín, en una proporción 1:2:2, respectivamente. El suelo empleado se recolectó en el Parque Nacional Zoquiapan, localizado a una altitud de $3350 \mathrm{~m}, 19^{\circ} 20^{\prime} 00^{\prime \prime} \mathrm{LN}$ y $98^{\circ} 42^{\prime} 00^{\prime}$ '
LO. Las semillas se sometieron a escarificación y esterilización superficial con peróxido de hidrógeno $\left(\mathrm{H}_{2} \mathrm{O}_{2}\right)$ a $30 \%$, durante $20 \mathrm{~min}$ y luego se lavaron con $1 \mathrm{~L} \mathrm{de}$ agua destilada estéril, tres veces.

El inóculo de hongos ECM utilizado se obtuvo a partir de esporomas adquiridos en mercados de la zona de influencia, en Ozumba y Chalco, Estado de México; estas estructuras se deshidrataron en una estufa (FELI® Aut. DGE 11524) a $36{ }^{\circ} \mathrm{C}$, durante $48 \mathrm{~h}$, para mantener la viabilidad de los propágulos. Después los esporomas deshidratados se molieron con un molino eléctrico, se pasaron a través de un tamiz de $1 \mathrm{~mm}$ (para homogeneizar el tamaño de las partículas), se depositaron en botes de plástico de $400 \mathrm{~mL}$ y se almacenaron en un refrigerador a $5{ }^{\circ} \mathrm{C}$, hasta su aplicación.

Como macetas se usaron recipientes de plástico (APB®) de $140 \mathrm{~cm}^{3}$ a los cuales se les agregó $150 \mathrm{~g}$ de la mezcla del sustrato, y de inmediato se sembraron tres semillas de cada especie de pino por recipiente; $35 \mathrm{~d}$ después se inocularon los hongos ECM. La segunda inoculación se efectuó 90 d después de la siembra. Cada planta se inoculó, en total, con $3 \mathrm{~cm}^{3}$ de esporomas molidos, los cuales contenían de $10^{6}$ a $10^{8}$ esporas, de acuerdo con evaluaciones efectuadas con un hematocitómetro (Neubauer®) ). Durante el experimento se probaron dos intervalos de humedad, 80 a 100 y 40 a 60 $\%$ de la capacidad máxima de retención de agua del sustrato (CMRAS). Para mantener dicha humedad se utilizó el método gravimétrico: se pesó el recipiente con el sustrato y la planta, según los tratamientos de humedad; cada $24 \mathrm{~h}$ se comprobó el peso del recipiente con una balanza granataria (OHAUS® de 2610 g y resolución de $0.1 \mathrm{~g}$ ), y se procedió a reponer el peso del agua perdida para volver al peso original.

Se aplicaron tres dosis de fertilización con el equivalente de 140,70 y $0 \mathrm{~kg}$ de $\mathrm{N} \mathrm{ha}^{-1}$, al usar como fuente $\mathrm{NH}_{4} \mathrm{NO}_{3}\left(\right.$ Meyer $\left.{ }^{\circledR}\right)$ ) con $35.5 \%$ de $\mathrm{N}$ (Fenn et al., 2003). La fertilización se dividió en cuatro aplicaciones en agua de riego: la primera parte se suministró 94 d después de la siembra, y las demás fueron escalonadas cada $15 \mathrm{~d}$. Las plantas se regaron con agua destilada durante $310 \mathrm{~d}$ y se les aplicó Captán® diluido, 2 g i.a. $\mathrm{L}^{-1}$ de agua de riego, para controlar algunos patógenos sin dañar a los inóculos.

Se utilizó un diseño experimental completamente al azar con arreglo de tratamientos factorial completo $2 \times 2 \times$ $2 \times 3 \times 4$, para analizar dos tipos de sustrato (estéril y no estéril), dos especies de pino ( $P$. patula y $P$. hartwegii), dos niveles de CMRAS (80 a 100 y 40 a $60 \%$ ), tres dosis de $\mathrm{N}\left(140,70\right.$ y $\left.0 \mathrm{~kg} \mathrm{ha}^{-1}\right)$, y cuatro tratamientos de 
hongos ECM (sin inoculación, Laccaria spp., Hebeloma spp. y Clavulina aff. cinerea), lo cual generó un total de 96 tratamientos; se tuvieron tres repeticiones para un total de 288 unidades experimentales, cada una constituida por una planta de pino.

Como variables de respuesta se midió: altura y diámetro de tallo en la base de cada planta, a 90, 180 y 310 d después de la siembra. En la última fecha las plantas se extrajeron de los recipientes y se colocaron en un recipiente con agua corriente para remover las partículas del sustrato. Después se contaron las raíces cortas mediante un microscopio estereoscópico (Zeiss $\left.\AA^{\circledR}\right)$ para las 288 plántulas de pino que constituyeron el experimento. Éstas se separaron en raíces cortas: $i$ ) micorrizadas, $i i)$ no micorrizadas, y iii) muertas. En las raíces micorrizadas se contabilizaron las que eran morfológicamente similares a las descritas para Laccaria spp. y Hebeloma spp., conforme a los criterios de Deemy (2008). Después se separó la raíz y la parte aérea de la planta y ambas partes se secaron en una estufa $($ Napco®mod. 620) con circulación de aire forzado a $70{ }^{\circ} \mathrm{C}$ durante $48 \mathrm{~h}$, para obtener el peso seco.

Con los datos de cada variable dependiente medida se realizó un análisis de varianza en el que se cuantificó la variación debida a tratamientos y sus interacciones. También se hizo una prueba de comparación de medias de Tukey $(\alpha=0.05 \%)$. Estos análisis se hicieron con el paquete estadístico SAS, ver. 9.0 (SAS Institute, 1999).

\section{RESULTADOS}

\section{Crecimiento}

Las variables de crecimiento de $P$. patula y $P$. hartwegii que resultaron más afectadas $(\mathrm{P} \leq 0.05)$ por los tratamientos fueron: peso seco del follaje, peso seco total y número de raíces cortas. También fueron afectadas $(\mathrm{P} \leq$ 0.05), con menor frecuencia, el peso seco de raíz, la altura y el diámetro de la planta (Cuadro 1).

Cuadro 1. Significancia de F en variables del crecimiento de Pinus patula y Pinus hartwegii, en diversos tratamientos de humedad, nitrógeno e inoculación ectomicorrízica en invernadero.

\begin{tabular}{|c|c|c|c|c|c|c|c|}
\hline Tratamientos & $\mathrm{gl}$ & PSF & PSR & PST & ALT & DIAM & NRC \\
\hline E & 1 & 0.21 & 0.54 & 0.66 & 0.73 & 0.29 & $<0.0001^{* *}$ \\
\hline $\mathrm{H}$ & 1 & $<0.0001^{* *}$ & $<0.0001^{* *}$ & $<0.0001^{* *}$ & $<0.0001^{* *}$ & $<0.0001^{* *}$ & $<0.0001^{* *}$ \\
\hline$P$ & 1 & $<0.0001^{* *}$ & $<0.0001^{* *}$ & $<0.0001 * *$ & $<0.0001 * *$ & $<0.0001 * *$ & $<0.0001^{* *}$ \\
\hline $\mathrm{N}$ & 2 & $0.03 *$ & 0.93 & 0.20 & 0.50 & 0.78 & 0.75 \\
\hline $\mathrm{E} \times \mathrm{H}$ & 1 & 0.21 & 0.55 & 0.27 & 0.35 & 0.10 & 0.56 \\
\hline$E \times P$ & 1 & 0.71 & 0.69 & 0.65 & 0.27 & 0.88 & 0.69 \\
\hline $\mathrm{H} \times \mathrm{P}$ & 1 & $0.0005^{* *}$ & $0.02 *$ & $0.0015^{* *}$ & $<0.0001 * *$ & 0.18 & $<0.0001^{* *}$ \\
\hline $\mathrm{H} \times \mathrm{I}$ & 3 & $<0.0001^{* *}$ & $<0.0001^{* *}$ & $<0.0001^{* *}$ & $0.0006^{* *}$ & 0.17 & $<0.0001^{* *}$ \\
\hline $\mathrm{H} \times \mathrm{N}$ & 2 & 0.31 & 0.55 & 0.36 & 0.31 & 0.45 & 0.20 \\
\hline$P \times I$ & 3 & $<0.0001 * *$ & $0.0006^{* *}$ & $<0.0001 * *$ & $<0.0001 * *$ & 0.40 & $0.0025 * *$ \\
\hline$P \times N$ & 2 & 0.07 & 0.40 & 0.14 & 0.62 & 0.34 & 0.27 \\
\hline I x N & 6 & $0.0048 * *$ & 0.27 & $0.05^{*}$ & 0.30 & 0.34 & $0.009 * *$ \\
\hline$E \times I \times N$ & 6 & $0.01 * *$ & $0.01 *$ & $0.01 * *$ & $0.02 *$ & $0.04 *$ & 0.99 \\
\hline $\mathrm{H} \times \mathrm{P} \times \mathrm{I}$ & 3 & $0.0038 * *$ & 0.06 & $0.01 * *$ & $0.0023^{* *}$ & 0.54 & $<0.0001^{* *}$ \\
\hline $\mathrm{H} \times \mathrm{P} \times \mathrm{N}$ & 2 & 0.65 & 0.77 & 0.66 & 0.22 & $0.0005^{* *}$ & 0.03 \\
\hline $\mathrm{H} \times \mathrm{I} \times \mathrm{N}$ & 6 & 0.07 & 0.18 & 0.11 & 0.18 & 0.38 & 0.56 \\
\hline$P \times I \times N$ & 6 & 0.18 & 0.73 & 0.65 & 0.65 & 0.20 & 0.50 \\
\hline $\mathrm{E} \times \mathrm{H} \times \mathrm{P} \times \mathrm{I}$ & 3 & 0.66 & 0.65 & 0.99 & 0.81 & 0.66 & $0.0018 * *$ \\
\hline $\mathrm{E} \times \mathrm{H} \times \mathrm{P} \times \mathrm{N}$ & 2 & 0.14 & 0.25 & 0.16 & 0.06 & 0.39 & 0.61 \\
\hline $\mathrm{H} \times \mathrm{P} \times \mathrm{I} \times \mathrm{N}$ & 6 & 0.14 & 0.73 & 0.35 & 0.30 & 0.28 & 0.75 \\
\hline $\mathrm{E} \times \mathrm{P} \times \mathrm{I} \times \mathrm{N}$ & 6 & $0.02 * *$ & $0.01 * *$ & $0.01 * *$ & 0.16 & 0.18 & 0.79 \\
\hline $\mathrm{E} \times \mathrm{H} \times \mathrm{I} \times \mathrm{N}$ & 6 & 0.56 & 0.38 & 0.57 & 0.40 & 0.34 & 0.72 \\
\hline ExHXPXIxN & 6 & 0.32 & 0.66 & 0.43 & $0.05^{*}$ & 0.70 & 0.16 \\
\hline
\end{tabular}

$\mathrm{E}=$ Esterilización del suelo; $\mathrm{H}=$ Humedad; $\mathrm{P}=$ Especie de pino; $\mathrm{I}=$ Inoculación con hongos ectomicorrízicos; $\mathrm{N}=$ Fertilización nitrogenada; PSF = Peso seco del follaje; PSR = Peso seco de la raíz; PST = Peso seco total; ALT = Altura de las plantas; DIA = Diámetro del tallo; NRC = Número de raíces cortas. 
En ambas especies de pino se observó que el régimen de humedad edáfica de 80 a $100 \%$ originó mayor crecimiento, en términos de peso seco de la planta, peso seco del follaje, peso seco de raíz, altura de planta, diámetro y producción de raíces cortas, en comparación con el régimen de 40 a $60 \%$ (Figura 1).

En la interacción entre humedad y especie de pino se presentaron diferencias $(\mathrm{P} \leq 0.05)$ para todas las

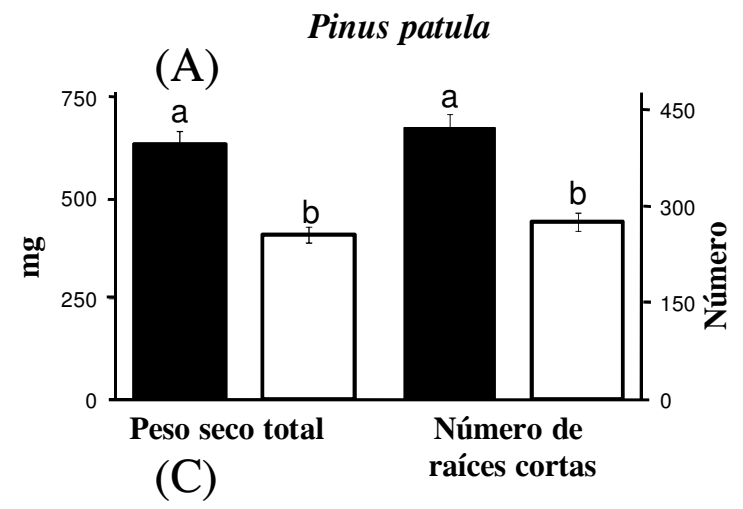

(E)

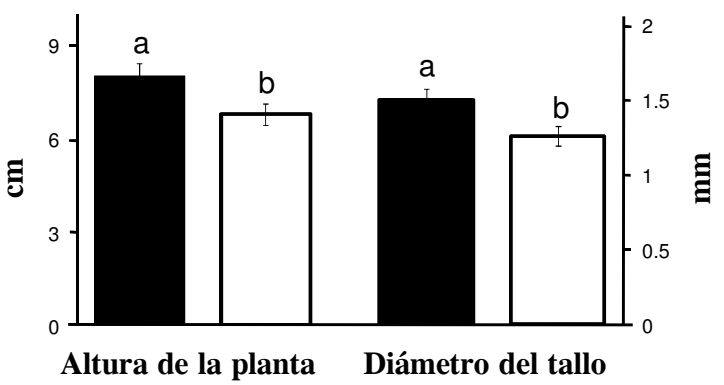

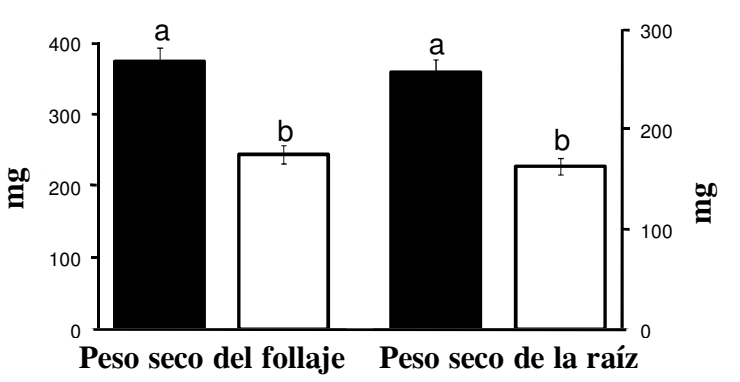

variables, excepto en diámetro del tallo (Cuadro 1). $P$. patula tuvo mayores valores de peso seco total, peso seco del follaje y raíz, y altura y diámetro del tallo en el régimen de 80 a $100 \%$ que en el régimen de 40 a $60 \%$, en plantas inoculadas con Hebeloma spp. En contraste, en las plantas de $P$. patula inoculadas con Laccaria spp., con Clavulina aff. cinerea y en las plantas no inoculadas, no hubo diferencias de crecimiento entre los dos regímenes de humedad.

\section{Pinus hartwegii}

(B)
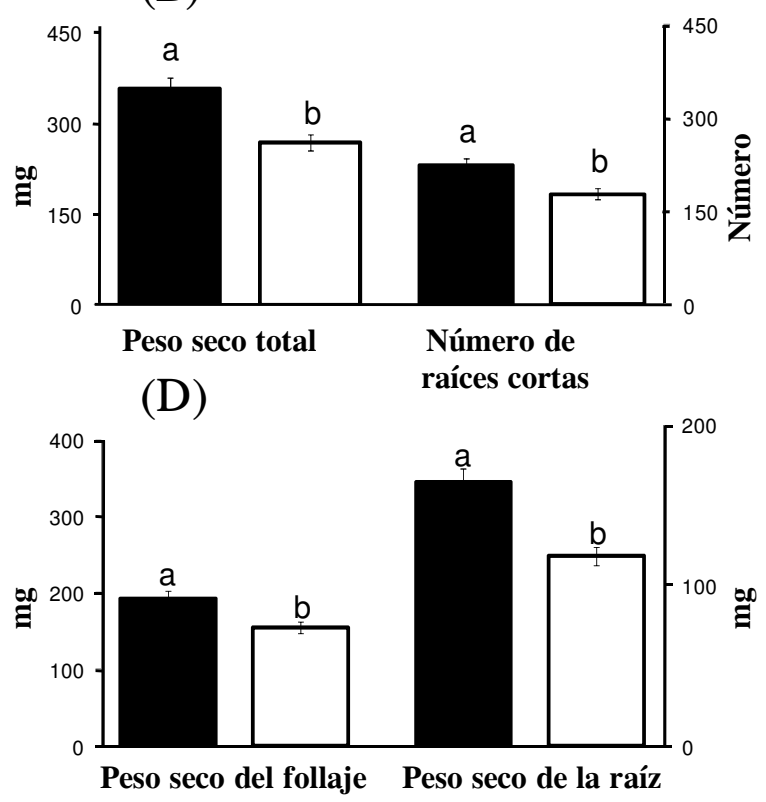

(F)

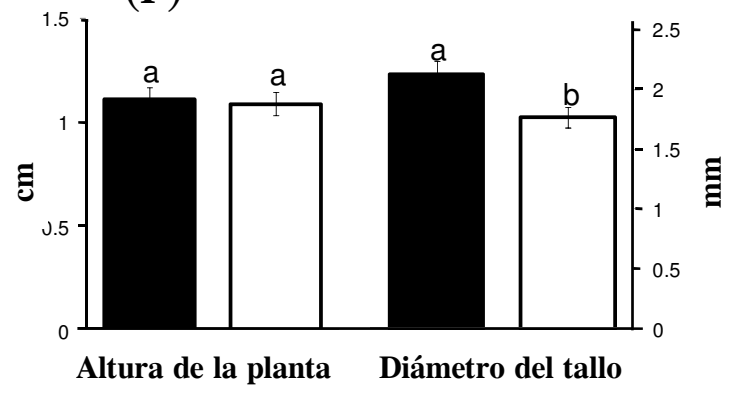

Figura 1. Promedios $(n=72)$ de peso seco total, número de raíces cortas, peso seco del follaje, peso seco de la raíz, altura y diámetro del tallo en árboles de Pinus patula $(\mathrm{A}, \mathrm{C}, \mathrm{E}) \mathrm{y}$ P. hartwegii $(\mathrm{B}, \mathrm{D}, \mathrm{F})$ crecidos en dos regímenes de humedad. Las barras negras corresponden al régimen de humedad que varió de 80 a $100 \%$ de la capacidad máxima de retención de agua del sustrato, y las barras blancas corresponden al régimen de humedad de 40 a $60 \%$ de dicha capacidad. Valores con la misma letra en cada variable y cada especie de pino son estadísticamente iguales (tStudent, 0.05). 
La producción de raíces cortas se incrementó en plantas inoculadas con Hebeloma spp. y en las inoculadas con Laccaria spp., pero no en plantas sin inocular o inoculadas con Clavulina aff. cinerea (Cuadro 2). En P. hartwegii sólo se observaron diferencias en peso seco total y peso seco de raíz en el régimen de 80 a $100 \%$, en comparación con el régimen de 40 a $60 \%$, en plantas inoculadas con Hebeloma spp.

En cuanto a los niveles de fertilización, en la mayoría de las variables evaluadas no se detectaron diferencias entre 0,70 y $140 \mathrm{~kg}$ de $\mathrm{N} \mathrm{ha}^{-1}$. Una excepción se registró en las plantas de $P$. patula inoculadas con Hebeloma spp., las cuales aumentaron el peso seco del follaje conforme se incrementó la aplicación de nitrógeno de 0 a 70 ó $140 \mathrm{~kg}$ de $\mathrm{N} \mathrm{ha}^{-1}$ (Cuadro 3). En la interacción entre humedad y nitrógeno no se detectaron variaciones significativas en las variables evaluadas (Cuadro 1), lo que parece indicar que la utilización del nitrógeno por la planta fue similar en los dos niveles de humedad.

Los pesos secos de raíz y parte aérea de $P$. patula y $P$. hartwegii (Figura 2) fueron mayores $(\mathrm{P} \leq 0.05)$ en las plantas inoculadas con Hebeloma spp., seguidas por las inoculadas con Laccaria spp., en comparación con las plantas sin inocular y las inoculadas, con Clavulina aff. cinerea, que no formaron ectomicorrizas en ninguna de las dos especies de pino.

Cuadro 2. Promedios $(\mathrm{n}=18)$ de peso seco, altura, diámetro y producción de raíces cortas en plantas de Pinus patula y Pinus hartwegii inoculadas o no con tres especies de hongos ectomicorrízicos en dos niveles de humedad de suelo.

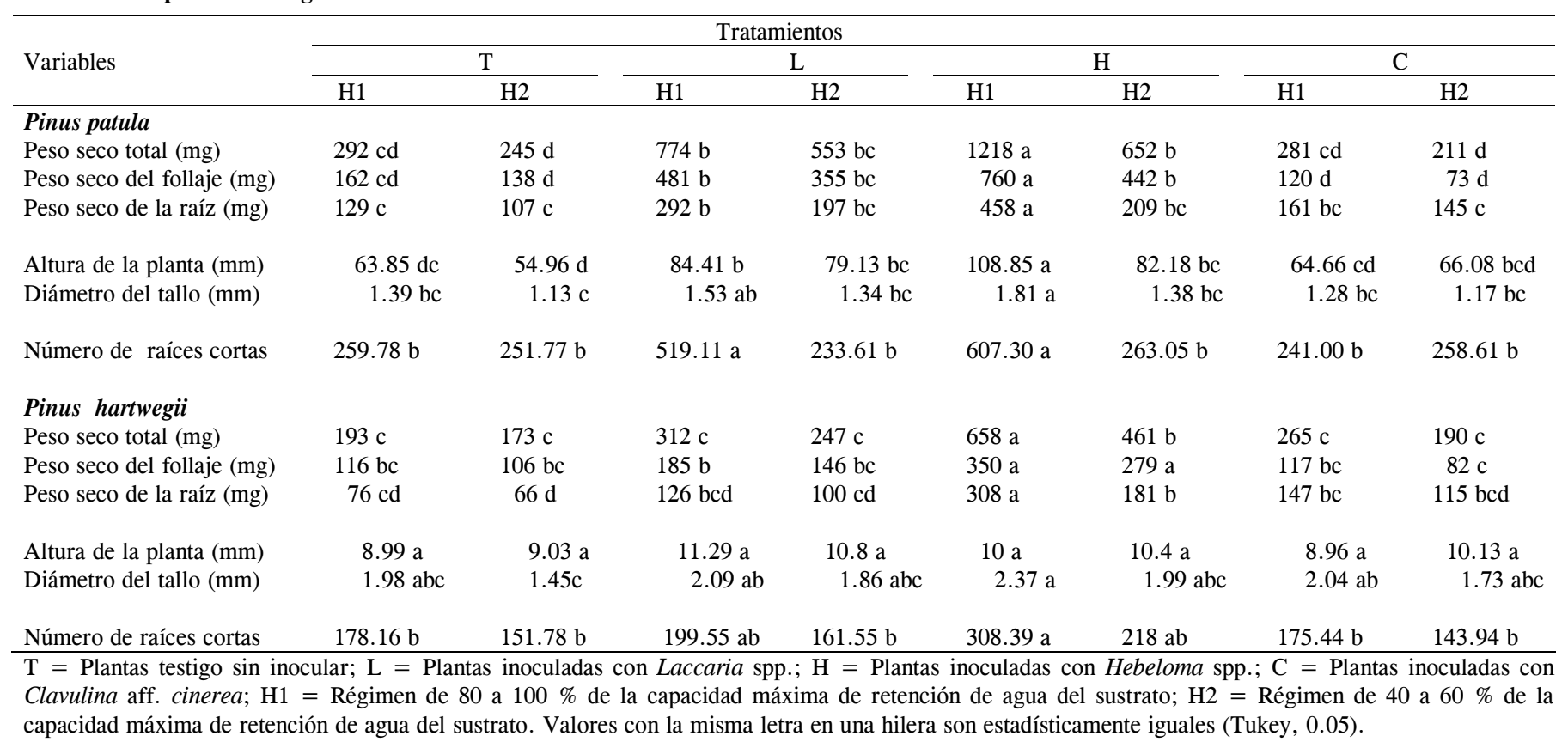
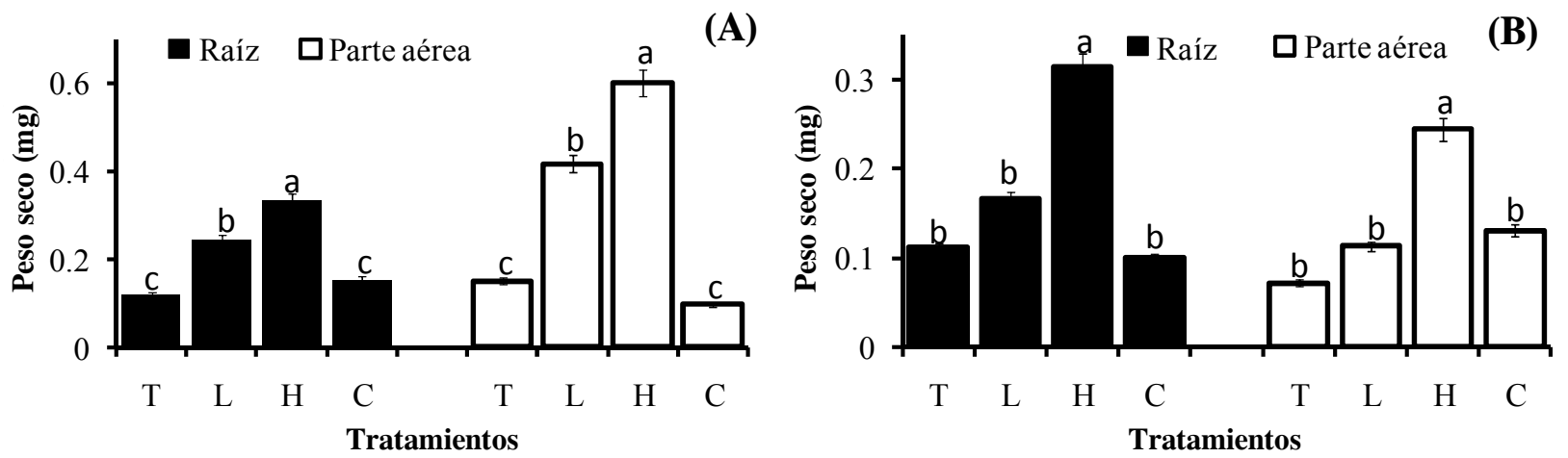

Figura 2. Plantas de Pinus patula (A) y Pinus hartwegii (B) inoculadas con tres hongos ectomicorrízicos comestibles (L = Laccaria spp., $\mathbf{H}=$ Hebeloma spp., $\mathrm{C}=$ Clavulina aff. cinerea) y plantas $\sin$ inocular $(\mathrm{T}=$ testigo). Los valores son promedios de 36 plantas. Promedios $(\mathrm{n}=36)$ con la misma letra en cada parte de las plantas, son estadísticamente iguales (Tukey, 0.05). Las líneas verticales sobre las barras representan el error estándar de la media. 


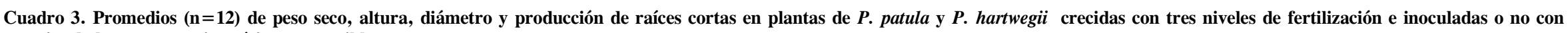
especies de hongos ectomicorrízicos comestibles.

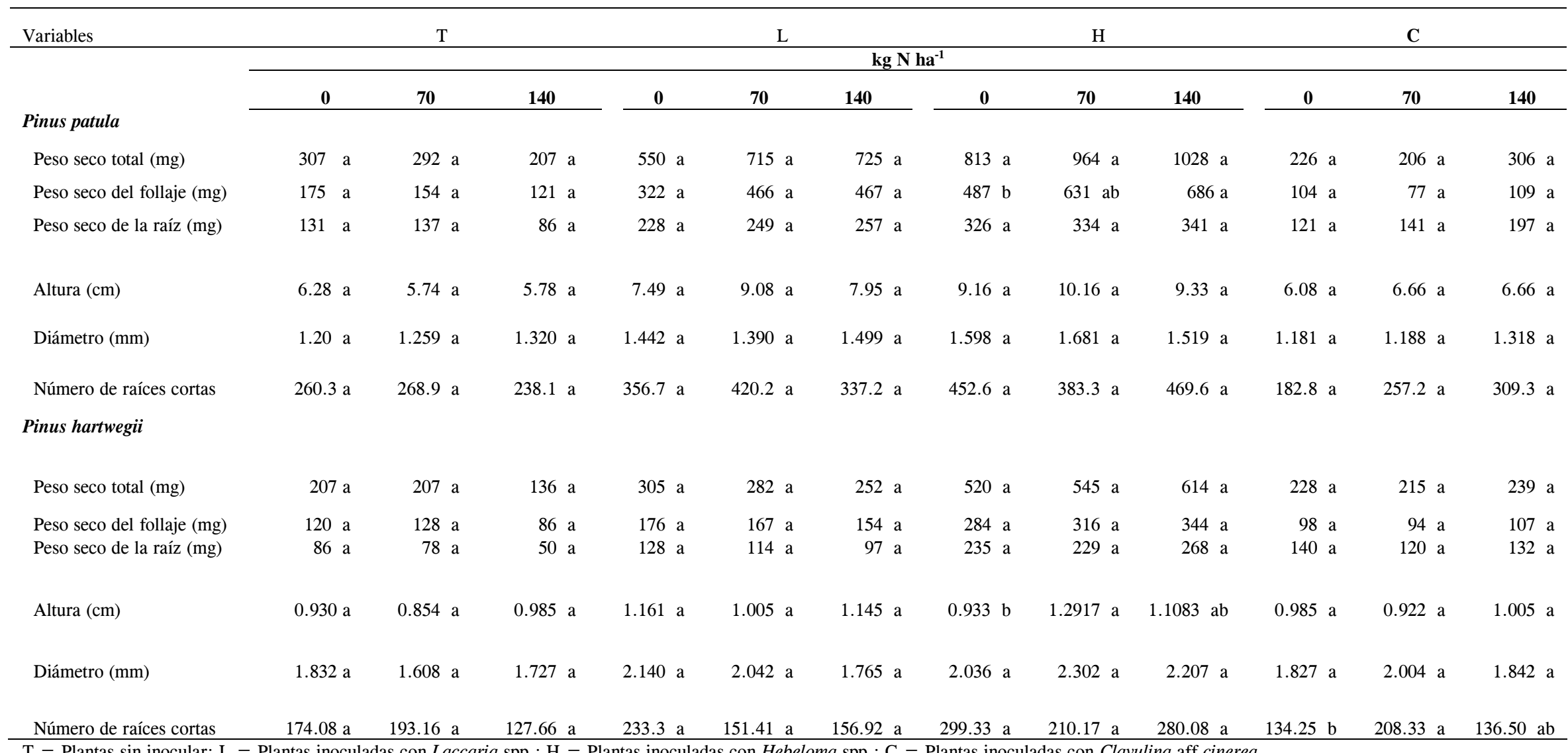

$\mathrm{T}=$ Plantas sin inocular; L = Plantas inoculadas con Laccaria spp.; H = Plantas inoculadas con Hebeloma spp.; C = Plantas inoculadas con Clavulina aff cinerea. 
En $P$. patula, desde los $90 \mathrm{~d}$ después de la inoculación se observó mayor altura $(\mathrm{P} \leq 0.05)$ en las plantas inoculadas con Hebeloma spp. y Laccaria spp., que en las plantas no inoculadas o inoculadas con Clavulina aff. cinerea. En $P$. hartwegii no hubo diferencias estadísticas en altura entre plantas inoculadas o sin inocular, incluso a 310 d después de la inoculación.

Entre los niveles de esterilización del sustrato no hubo diferencias $(\mathrm{P} \leq 0.05)$ en crecimiento, altura, diámetro del tallo, peso seco del follaje, peso seco de raíz y peso seco total, al comparar las plantas crecidas en sustratos estériles $v s$. sustratos no estériles.

\section{Colonización ectomicorrízica}

En $P$. patula el número total de raíces cortas colonizadas por Laccaria spp. fue más alto que en los inoculados con Hebeloma spp. a 310 d después de la siembra, independientemente de la condición de esterilización del suelo (Figura 3A), mientras que en $P$. hartwegii se observó un escaso o nulo número de raíces cortas colonizadas con Hebeloma spp. y Laccaria spp. (Figura 3B). La morfoanatomía aquí encontrada para Laccaria spp. y Hebeloma spp. fue similar a la reportada por Deemy (2008) para dichos géneros.

\section{DISCUSIÓN}

La micorrización con Hebeloma spp. en el régimen de humedad edáfica de 80 a $100 \%$ estimuló el crecimiento de $P$. patula e incrementó el número de raíces cortas, en comparación con las desarrolladas en el régimen de 40 a $60 \%$. Según Muhsin y Zwiazek (2000), el micelio extrarradical y la red hifal apoplástica (transporte extracelular de los iones hasta la endodermis) de las raíces ectomicorrizadas juegan un importante papel en la absorción y transporte de agua a la planta huésped. Estos mismos autores agregan que las hifas de hongos ectomicorrízicos están involucradas en el incremento de la conductividad hidráulica de las raíces, al reducir la resistencia del flujo de agua del apoplasto. A este respecto Egerton-Warburton et al. (2007) mencionaron que el movimiento de agua por redes comunes micorrízicas es potencialmente importante para la supervivencia de las plantas durante la sequía. Runion et al. (1997) reportaron un alto porcentaje de raíces cortas colonizadas por ectomicorrizas en Pinus palustris Mill. bajo un adecuado suministro de agua, en comparación con el tratamiento en el que hubo falta de agua y las plantas tuvieron menor longitud de raíz y baja micorrización.

En el presente trabajo se observó una reducción en el desarrollo de raíces cortas en el régimen de humedad 40 a $60 \%$ en ambas especies de árboles estudiadas. Este fenómeno también fue observado en campo, en Oaxaca, México, en $P$. oaxacana Mirov por Valdés et al. (2006), quienes registraron una disminución de $60 \%$ del total de raíces cortas y de la biomasa de raíces ectomicorrizadas durante un año con sequía, en comparación con un año sin sequía.
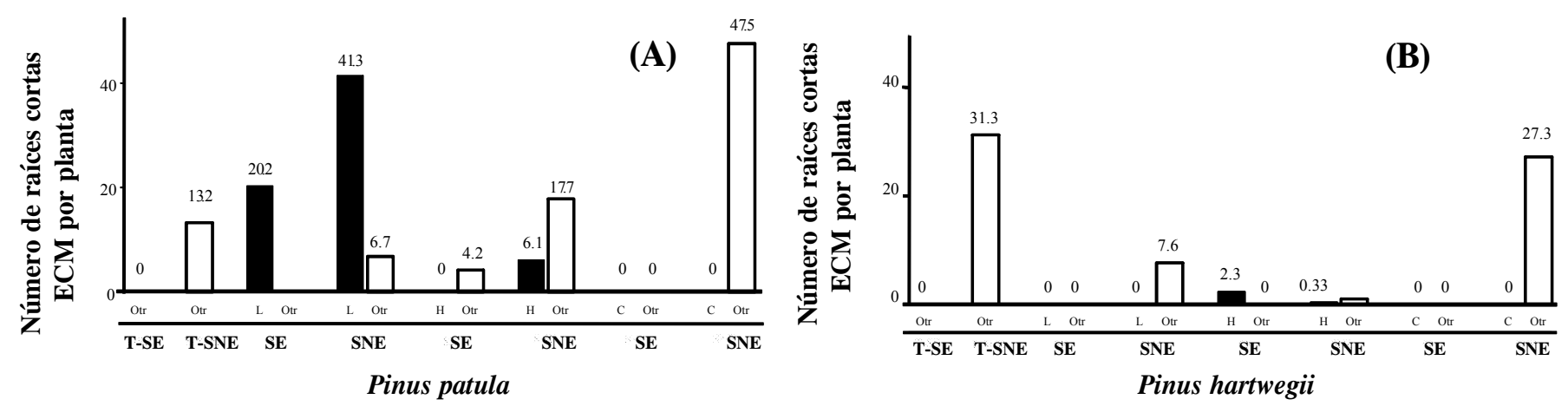

Figura 3. Promedios $(\mathrm{n}=18)$ del número de raíces cortas ectomicorrizadas (ECM) en plantas de Pinus patula (A) y Pinus hartwegii (B) inoculadas con tres hongos ectomicorrízicos $(\mathrm{L}=$ Laccaria spp., $\mathrm{H}=$ Hebeloma spp., $\mathrm{C}=$ Clavulina aff. cinerea $)$ y plantas sin inocular $(\mathrm{T}=$ testigo). $\mathrm{SE}=$ Suelo estéril; SNE = Suelo no estéril; Otr = Otros hongos ectomicorrízicos distintos a los inoculados. Las barras llenas, con patrón inclinado y huecas corresponden al número de raíces cortas ECM con la morfología característica de Laccaria spp., Hebeloma spp. y hongos ECM distintos de los inoculados, respectivamente. 
En el presente experimento, el número de raíces cortas en las plantas inoculadas (Hebeloma spp. y Laccaria spp.) en el régimen de 80 a $100 \%$ fue mayor que en las plantas no inoculadas. Esto puede deberse a que las plantas colonizadas con hongos ECM tienen una mayor tasa respiratoria en la raíz que las plantas sin colonizar y, por tanto, tienen mayores requerimientos de carbono, lo cual origina un incremento en la fotosíntesis, como reportaron Domínguez-Núnez et al. (2004). También se ha señalado que las hifas de hongos ectomicorrízicos son transportadores eficientes de agua (Egerton-Warburton et $a l .$, 2007) y de esta forma potencian la exploración del agua en el suelo, incrementan la asimilación de $\mathrm{CO}_{2}$ (Wright et al., 2000) y el crecimiento de las plantas (Wullschleger et al., 2002).

Los mecanismos mediante los cuales los hongos ECM pueden mejorar la absorción de agua en las plantas son: $i$ ) a través de las redes hifales de micelio externo, las cuales incrementan el área de exploración de la raíz y transportan agua que de otra forma no estaría disponible para las raíces; y ii) mediante la modulación de los requerimientos hídricos de las plantas asociadas, al mejorar su nutrición y así reducir su demanda por transpiración (Marjanovic y Nehls, 2008). Las diferencias en la respuesta de las plantas para mejorar sus relaciones hídricas por efecto de las micorrizas, dependen de las especies de hongos ECM asociados con las raíces (Park et al., 1983), debido a las diversas propiedades morfológicas y anatómicas particulares de cada especie de hongo ECM que pueden afectar diferencialmente la absorción de agua en las plantas asociadas. Por ejemplo, la presencia de rizomorfos aquí, observados en Hebeloma spp; pero no en Laccaria spp.

Los rizomorfos son cordones miceliales macroscópicos, con hifas arregladas de forma paralela, que con frecuencia tienen hifas centrales huecas alargadas rodeadas de hifas vivas más pequeñas (Agerer, 2001; Brownlee et al., 1983). Se ha demostrado que estas estructuras facilitan el transporte de agua del suelo donde crecen las hifas hacia las raíces ECM y las plantas hospederas. Adicionalmente, se ha propuesto que dicho trasporte de agua se efectúa a través de las hifas alargadas huecas dentro de los rizomorfos (Duddridge et al., 1980; Brownlee et al., 1983), y que la hidrofobia de los rizomorfos puede reducir sustancialmente las pérdidas de agua cuando es transportada en largas distancias (Agerer, 2006). Se ha sugerido además que: $i$ ) el movimiento de agua por el micelio de redes micorrízicas que conectan raíces de plantas de la misma o distintas especies vegetales es potencialmente importante para la supervivencia de las plantas contra la sequía, y ii) las características específicas individuales de las diversas especies de hongos ECM puede ser un componente importante de este mecanismo (Egerton-Warburton et al., 2007).

En $P$. patula inoculado con Hebeloma spp. y fertilizado con $140 \mathrm{~kg} \mathrm{~N} \mathrm{ha}^{-1}$ se incrementó el peso seco del follaje, en comparación con el tratamiento sin aplicación de N. En $P$. hartwegii inoculado con Hebeloma spp. se observó un aumento significativo en altura de la planta y en número de raíces cortas, respectivamente, al aplicar $70 \mathrm{~kg} \mathrm{~N} \mathrm{ha}^{-1}$. Esto probablemente se debió a que la simbiosis micorrízica es capaz de mejorar la aportación de nutrimentos a la planta, al incrementar las formas disponibles de éstos, especialmente P y N (Sylvia et al., 1999). Una explicación puede ser que las hifas del hongo utilizan su capacidad ecofisiológica para absorber agua y adquirir N (EgertonWarburton et al., 2003).

Sin embargo, no se encontró efecto negativo al aplicar $140 \mathrm{~kg} \mathrm{~N} \mathrm{ha}^{-1}$ en ninguna de las variables estudiadas. Con relación al efecto del nitrógeno atmosférico sobre las ectomicorrizas se ha reportado una declinación en la abundancia de micorrizas con altas dosis de nitrógeno (Carfrae et al., 2006). Arnebrant (2004) reportó un efecto negativo del $\mathrm{N}$ en el crecimiento de micelio en plántulas de pino fertilizadas con dos diferentes fuentes, sulfato de amonio [ $\left.\left(\mathrm{NH}_{4}\right)_{2} \mathrm{SO}_{4}\right]$ y nitrato de amonio $\left[\mathrm{NH}_{4} \mathrm{NO}_{3}\right]$, e inoculadas con cinco cepas de hongos: dos aislamientos de Paxillus involutus (Batsch) Fr., uno de Suillus bovinus (Pers.) Roussel y dos ectomicorrizas no identificadas.

Diversas investigaciones han demostrado que los esporomas y las comunidades subterráneas de hongos ECM son afectados negativamente como consecuencia del depósito de $\mathrm{N}$ atmosférico (Lilleskov et al. 2001; Peter y Ayer, 2001; Wallenda y Kottke, 1998). Sin embargo, en la presente investigación la colonización ECM por Laccaria spp. y Hebeloma spp. no resultó afectada por la fertilización. Es importante señalar que: i) sólo se evaluó la colonización ECM al final del experimento, y ii) en general, las colonizaciones micorrízicas encontradas fueron bajas en todos los tratamientos, como se señaló anteriormente.

En cuanto a las características morfológicas y anatómicas, las raíces micorrizadas correspondieron a dos géneros de hongos ECM inoculados, Hebeloma spp. y Laccaria spp.; en cambio, en Clavulina aff. cinerea no se encontraron evidencias de colonización ectomicorrízica en las raíces de las plantas evaluadas, a pesar de que esta especie se ha reportado como ectomicorrízica en bosques de Abies spp. (Ivanov, 2005). Esto posiblemente se deba a la naturaleza de los esporomas utilizados para la preparación del inoculante, ya que Clavulina aff. cinerea tiene esporomas con numerosas ramas cilíndricas surcadas 
longitudinalmente y con esporas expuestas, las cuales pueden perderse con facilidad durante la recolección y el secado. Las características morfológicas y anatómicas de las raíces micorrizadas de Hebeloma spp. y Laccaria spp. aquí encontradas, resultaron similares a las reportadas por Deemy (2008).

A pesar de los conspicuos incrementos de biomasa registrados en $P$. patula y $P$. hartwegii, como consecuencia de la inoculación con hongos ectomicorrízicos, se registró una baja colonización ectomicorrízica. Un fenómeno similar lo encontró Cripps (2001), al inocular Populus tremuloides Michx. con diversos hongos ECM; este autor atribuyó este fenómeno a una alta eficiencia en la transferencia de nutrimentos con un bajo número de raíces ectomicorrizadas, o a la liberación sustancial de fitohormonas, como el ácido indolacético, en el estadio inicial de la micorrización.

\section{CONCLUSIONES}

El crecimiento vegetal de las especies de pino resultó afectado $(\mathrm{P} \leq 0.05)$ por los dos regímenes de humedad estudiados, en magnitud que varió entre las especies de los hongos ectomicorrízicos inoculados y entre las especies de plantas. Las plantas de $P$. patula inoculadas con Hebeloma spp. presentaron mayores pesos secos total, del follaje y la raíz, así como mayor altura de planta y diámetro de tallo, en comparación con las plantas inoculadas con Laccaria spp., Clavulina aff. cinerea o sin inocular, cuando crecieron en el régimen de 80 a $100 \%$ de la humead edáfica, comparado con el régimen de 40 a $60 \%$. Las colonizaciones micorrízicas encontradas al final del experimento fueron bajas en todos los tratamientos y no resultaron afectadas por la fertilización nitrogenada.

\section{AGRADECIMIENTOS}

Al CONACyT por la beca doctoral al primer autor. A la SEMARNAT y CONACyT por el financiamiento otorgado en el proyecto SEMARNAT-CONACyT-200401-45: "Los hongos silvestres comestibles del Parque Nacional Izta-Popo, Zoquiapan y Anexos". La caracterización morfo-anatómica, identificación y cuantificación de las ectomicorrizas se realizó en el Laboratorio de Recursos Genéticos en Hongos del Campus Montecillo, bajo la asesoría del Dr. Luis Villarreal Ruiz.

\section{BIBLIOGRAFÍA}

Agerer R (2001) Exploration types of ectomycorrhizae. A proposal to classify ectomycorrhizal mycelia systems according to their patterns of differentiation and putative ecological importance. Mycorrhiza 11:107-114.
Agerer R (2006) Fungal relationships and structural identity of their ectomycorrhizae. Mycol. Progress 5: 67-107.

Alvarado-Rosales D, T Hernandez-Tejeda (2002) Decline of sacred fir in the Desierto de los Leones National Park. Urban Air Pollution and Forests: Resources at Risk in the Mexico City Air Basin. In: M E Fenn, L I de Bauer, T Hernandez T (eds). Ecological Studies 156. Springer-Verlag. New York. pp:243-260.

Arnebrant K (2004) Nitrogen amendments reduce the growth of extramatrical ectomycorrhizal mycelium. Mycorrhiza 5:7-15.

Bogeat-Triboulot M B, F Bartoli, J Garbaye, R Marmeisse, D Tagu (2004) Fungal ectomycorrhizal community and drought affect root hydraulic properties and soil adherence to roots of Pinus pinaster seedlings. Plant Soil 267:213-223.

Brownlee C, J A Duddridge, A Malibari, D J Read (1983) The estructure and function of mycelia systems of ectomycorrhizal roots with special reference to their role in forming inter-plant connections and providing pathways for assimilate and water transport. Plant Soil 71:433-443.

Campos J L (1993) Claves para la determinación de los pinos mexicanos. Universidad Autónoma Chapingo. Serie de Apoyos Académicos 23. Chapingo, México. $70 \mathrm{p}$.

Carfrae J A, K R Skene, L J Sheppard, K Ingleby, A Crossley (2006) Effects of nitrogen with and without acidified sulphur on an ectomycorrhizal community in a Sitka spruce (Picea sitchensis Bong. Carr) forest. Environ. Pollution 196:159-171.

Cripps C L (2001) Mycorrhizal Fungi of Aspen Forests: Natural Occurrence and Potential Applications. Sustaining aspen in western landscapes. In: Symp. Proc. June 13-15, 2000, Grand Junction, Colorado. USDA Forest Service. Rocky Mountain Research Station Fort Collins, CO, USA. pp:285-298.

Davies F T, S E Svenson, J C Cole, L Phavaphutanon, S A Duray, V Olalde-Portugal, C E Meier, S H Bo (1996) Non-nutritional stress acclimation of mycorrhizal woody plants exposed to drought. Tree Physiol. 16:985-993.

Deemy (2008) Characterization and determination of ectomycorrhizae. (www.deemy.de) (consultada el 15 de abril de 2009)

Domínguez-Núñez J A, R Planelles, J A Rodríguez-Barreal, J A Saiz de Omeñaca (2004) Influencia de la micorrización con trufa negra (Tuber melanosporum) en el crecimiento, intercambio gaseoso y nutrición mineral de plántulas de Pinus halepensis. Sist. Rec. For. 13:317-327.

Duddridge J A, A Malibari, D J Read (1980) Structure and function of mycorrhizal rhizomorphs with special reference to their role in water transport. Nature 283:834-836.

Dvorak W S, G R Hodge, J E Kietzka, F Malan, L F Osorio, T K Stanger (2000) Pinus patula. In: Conservation and Testing of Tropical and Subtropical Forest Tree Species by the CAMCORE Cooperative, College of Natural Resources, NCSU. Raleigh, NC, USA. pp:149-173.

Egerton-Warburton L M, J I Querejeta, M F Allen (2007) Common mycorrhizal networks provide a potential pathway for the transfer of hydraulically lifted water between plants. J. Exp. Bot. 6:14731483.

Egerton-Warburton L M, R C Graham, K R Hubbert (2003) Spatial variability in mycorrhizal hypae and nutrient and water availability in soil-weathered bedrock profile. Plant Soil 249:331342.

Fenn M E, J S Baron, E B Allen, H M Rueth, K R Nydick, L Geiser, W D Bowman, J O Sickman, T Meixner, D W Johnson, P Neitlich (2003) Ecological effects of nitrogen deposition in the Western United States. BioScience 53:404-420.

Ivanov D M (2005) Mycobionts of ectomycorrhizal of Picea abies in bilberry spruce forest. Mykol. I Fitopatol. 39:41-47.

Leake J, J David J, D Damian, M Gemma, B Lynne (2004) Networks of power and influence: The role of mycorrhizal mycelium in controlling plant communities and agroecosystem functioning. Can. J. Bot. 82:1016-1045. 
Lilleskov E A, T J Fahey, G M Lovett (2001) Ectomycorrhizal, fungal aboveground community change over an atmospheric nitrogen deposition gradient in Alaska. Ecol. Appl. 11:397-410.

Marjanovic Z, U Nehls (2008) Ectomycorrhiza and Water Transport. Mycorrhiza. In: A Varna Heidelberg (ed). Springer-Verlag Berlin. pp:149-159.

Muhsin T M, J J Zwiazek (2000) Ectomycorrhyzas increase apoplastic water transport and root hydraulic conductivity in Ulmus americana seedlings. New Phytol. 153:153-158.

Nilsen P, I Børja, H Knutsen, R Brean (1998) Nitrogen and drought effects on ectomycorrhizae of Norway spruce [Picea abies L.(Karst.)]. Plant Soil 198:79-184.

Paoletti E, B Andrzej, A Chris, A Algirdas, F Marco, G Nancy, G G Madeleine, I John, J Dale, K Dave, L Jesada, M Rainer, M Steven, S Gerhard Müller, M Robert, P Kevin (2007) Impacts of Air Pollution and Climate Change on Forest EcosystemsEmerging Research Needs. Sci. World J. 7:1-8.

Park J L, R G Linderman, C H Black (1983) The role of ectomycorrhyzas in drought tolerance of Douglas fir seedlings. New Phytol. 95:83-95.

Peter M, S E Ayer (2001) Nitrogen addition in a Norway spruce stand altered macromycete sporocarp production and below-ground ectomycorrhizal species composition. New Phytol. 149:311-325.
Runion GB, RJ Mitchell, H H Rogers, S A Prior, T K Counts (1997) Effects of nitrogen and water limitation and elevated atmospheric $\mathrm{CO}_{2}$ on ectomycorrhiza of longleaf pine. New Phytol. 137:681689.

SAS Institute (1999) SAS User's Guide, ver. 9.0. SAS Institute. Cary, NC, USA. $921 \mathrm{p}$.

Sylvia D, J Fuhrmann, P Hartel, D Zuberer (1999) Principles and Applications of Soil Microbiology. Prentice Hall. N J, USA. 550 p.

Valdés M, H Asbjornsen, M C Gómez, M Juárez, K A Vogt (2006) Drought effects on fine-root and ectomycorrhizal-root biomass in managed Pinus oaxacana Mirov stands in Oaxaca, Mexico. Mycorrhiza 16:117-124.

Wallenda T J, I Kottke (1998) Nitrogen deposition and ectomycorrhizal . New Phytol. 139:169-187.

Wright D P, J D Scholes, D J Read, S A Rolfe (2000) Changes in carbon allocation and expression of carbon transporter genes in Betula pendula Roth. colonized by the ectomycorrhizal fungus Paxillus involutus (Batsch) Fr. Plant Cell Environ. 23:39-49.

Wullschleger S D, T J Tschaplinki, R J Norby (2002) Plant water relations at elevated $\mathrm{CO}_{2}$ implications for water-limited environments. Plant Cell Environ. 25:319-331. 